\title{
Critical particle size where the Stokes-Einstein relation breaks down
}

\author{
Zhigang Li \\ Department of Mechanical Engineering, The Hong Kong University of Science and Technology, Clear Water Bay, Kowloon, Hong Kong
}

(Received 30 July 2009; revised manuscript received 20 September 2009; published 10 December 2009)

\begin{abstract}
The validity of the Stokes-Einstein (SE) relation for particle diffusion in the nano- and molecular scales has attracted much interest, but the results in the literature are controversial. In this work, it is shown that there exists a critical particle size where the SE relation breaks down by comparing particle transport in the macroand molecular scales. Using molecular-dynamics simulations, we study the critical size and find that the van der Waals force plays an important role in particle diffusion as the particle size approaches molecular scale. Due to the limitations of computing facilities, we could not find exactly where the critical particle size is, but the simulation results qualitatively predict that this critical size is of a few nanometers.
\end{abstract}

DOI: 10.1103/PhysRevE.80.061204

PACS number(s): 66.10.cg, 47.57.eb, 05.40.Jc

\section{INTRODUCTION}

Particle diffusion in fluids is an old scientific problem and of great importance in various studies, including chemistry, physics, material synthesis, and many areas in engineering [1-5]. The classical Stokes-Einstein (SE) relation relates the diffusion coefficient $D$ of a Brownian sphere in a fluid to the temperature $T$ and drag coefficient $\alpha$ through

$$
D=\frac{k T}{\alpha},
$$

where $k$ is the Boltzmann constant and $\alpha$ is defined by the Stokes law

$$
F=-\alpha V,
$$

where $F$ is the drag force on the sphere moving with a relative velocity $V$ to the fluid. For slip and stick boundary conditions, $\alpha=4 \pi \mu R$ and $6 \pi \mu R$, where $\mu$ is the viscosity of the fluid and $R$ is the radius of the sphere. Equation (1) can be derived from the Langevin equation if the linear relationship in Eq. (2) holds [6]. For macroscale spherical particles, Eqs. (1) and (2) have strong theoretical background and have been widely accepted $[3,4,7]$. However, with the advances in nanotechnology, the classical transport theories face challenges because some microscopic parameters, such as molecular interactions, which are not considered in the traditional treatments, may play important roles in particle transport [8-10]. Although some works have shown that the Stokes law and SE relation are valid for small solutes [11-13], many theoretical and experimental studies have found that the classical theories break down in many situations in the nano- and molecular scales $[9,10,14]$. As will be discussed later, there exists a critical value in the nanometer scale for the particle size, below which Eq. (1) becomes invalid. To clear up the confusions about the validity of the SE relation, it is necessary to investigate this critical value. This is also important for practical applications.

The validity of the SE relation for small particles has been of interest for many years. Both numerical and experimental works have been carried out to test the applicability of the classical transport theories to particles in the nano and molecular scales [11-13,15-25]. Experimentally it is difficult to investigate the effects of microparameters on the particle dif- fusion. Most of the previous works employ the method of molecular-dynamics (MD) simulation because it provides a flexible way to change the particle size, mass, and particlefluid interactions. However, the results appear to be controversial due to the difference in the simulation models. In most of the simulations, the particles do not have internal structures and the thermal vibrations of constituent atoms are not involved [15,16,19-24]. It has been shown that the thermal motion of the atoms in the particle affects the energy accommodation of the particle and is the major reason for fluid adsorption on the particle surface [8], which may lead to stick boundary conditions. Therefore, it is inappropriate to study nanoparticle transport by treating the particle as a hard sphere. In some other works, the internal structures of the particle are considered, but there are still discrepancies in the results $[13,18]$. This might be caused by different values of certain parameters used in the simulations. It is also noted that in the previous simulations, the effects of finite system size on the particle diffusivity are not considered. Hence, the validity of the SE relation in the nano- and molecular scales requires further investigations.

In this work, we show that there exists a critical size for the particle, above which Eq. (1) is valid, i.e., the diffusivity of a particle only depends on the viscosity of the fluid and the particle size given the temperature. When the particle size is smaller than this critical value, the van der Waals force tends to be important and has to be considered in understanding the particle transport in liquids. We shall focus our attention on the qualitative existence and prediction rather than the quantitative evaluation of the critical size. Furthermore, we only consider the particle transport in simple fluids because the breakdown of the classical transport theories in complex fluids, such as polymeric liquids, is caused by the remarkable change in the physical properties of the fluids instead of the van der Waals force [24,25]. Due to the limitations of the computing facilities, we cannot find the exact critical size, but detailed MD simulations indicate that this critical size is of a few nanometers.

\section{EXISTENCE OF CRITICAL PARTICLE SIZE}

Gas kinetic theory predicts that the van der Waals force is critical for nanoparticle transport in gases [9]. For particle 
transport in liquids, the van der Waals force may also play important roles when the particle size is in the nano- or smaller scale. This can be indicated by comparing the two limiting cases: macroparticle transport and molecular diffusion. For a macroparticle moving in a liquid, the diffusivity of the particle is given by the SE relation, i.e., it depends on the particle size $R$ and fluid viscosity $\mu$ only given the temperature. However, if the particle size shrinks down to molecular scale, the diffusion coefficient of the particle or molecule cannot be determined by the particle size and fluid viscosity without knowing the material of the particle. For example, the size ratio of $\mathrm{Kr}$ to $\mathrm{Ar}$ is about 1.05 [26], but the diffusion coefficient of $\mathrm{Kr}$ is about 3 times larger than that of Ar in oil [27]. In the molecular scale, some microparameters, such as the particle-fluid interaction parameters, may dominate and affect the particle dynamics. These two limiting cases indicate that the diffusivity converges from microparameters dependence to the $S E$ relation when the particle size changes from molecular to macroscales. Equivalently, it can be argued that there exists a critical particle size between the molecular and macroscales, below which the microparameters start to play a role and the SE relation generally becomes invalid.

Experimentally, it is difficult to find the critical particle size. However, based on the theoretical analysis of particle dynamics in rarefied gases [9], it is possible to roughly estimate where the critical size is without intensive explorations. For particle transport in rarefied gases, gas kinetic theory shows that the van der Waals force is inconsequential when the particle is larger than $\sim 30 \mathrm{~nm}$ in diameter [9]. For gasparticle interactions, the collisions between the gas molecules and particle dominate the momentum transfer and the particle dynamics. For particle transport in liquids, however, it is inappropriate to account for the momentum transfer by liquid-particle collisions because the collisions are greatly affected by the closely packed liquid molecules in the neighborhood. In this case, the transport properties of the particle are determined by the viscosity of the liquid and the van der Waals force should be much less important than the cases of particle motion in gases. Therefore, the critical size for particle transport in liquids should be much smaller than $30 \mathrm{~nm}$. If so, molecular-dynamics simulations can be used to calculate the diffusion coefficient of nanoparticles and subnanoclusters in liquids to generally predict the critical size.

\section{SIMULATION METHODS}

In a typical MD simulation system considered in this work, a solid particle is diffusing in a cubic simulation box, which is filled with liquid molecules. The diffusion coefficient of the particle is measured as the particle size is varied. The liquid is described by the Lennard-Jones (LJ) 12-6 potential function $U(r)=4 \varepsilon\left[(\sigma / r)^{12}-(\sigma / r)^{6}\right]$, where $r$ is the separation between a pair of molecules, $\sigma$ is the collision diameter, and $\varepsilon$ is the binding energy. The values for $\sigma$ and $\varepsilon$ are set to be $3.395 \AA$ and $116.8 \mathrm{~K}$, which correspond to $\mathrm{Ar}$ [28]. The solid particle is initially constructed by keeping all the atoms in a sphere of radius $R$ from a face-centered cubic structure with lattice constant equal to $4.08 \AA$, which corre- sponds to Ag. To consider the thermal motion of the atoms in the particle, a potential function is needed to calculate the interatomic interactions. The LJ potential function can be an option. It is simple and less time consuming. Another potential is the tight-binding potential function [8,29], which has been well tested and widely accepted for some transition metals, such as Ag and Au. It works well for considering the thermal vibrations of the atoms in the particle $[8,30]$. In particular, this potential can prevent the nanoparticles or clusters from being dissolved in the fluid if the temperature is not very high. To catch the fundamental physics taking place at the fluid-wall interface, we choose the tight-binding potential to model the solid particle. The interactions between the liquid molecules and particle atoms are also calculated through the LJ potential. To study the material dependence of the diffusion coefficient, the liquid-particle binding energy $\varepsilon_{\mathrm{LP}}$ is artificially changed with a wide range while $\sigma_{\mathrm{LP}}$ is chosen to be the same as that for Ar-Ar interaction. The mass of the particle atoms is set to be the same as that of Ar. The potential is truncated at $10.21 \AA$ and Newton's equations are integrated with Beeman's algorithm [8]

$$
\begin{aligned}
\mathbf{r}_{i+1} & =\mathbf{r}_{i}+\mathbf{v}_{i} \Delta t+\left(\Delta t^{2} / 6\right)\left(4 \mathbf{a}_{i}-\mathbf{a}_{i-1}\right) \\
\mathbf{v}_{i+1} & =\mathbf{v}_{i}+(\Delta t / 6)\left(2 \mathbf{a}_{i+1}+5 \mathbf{a}_{i}-\mathbf{a}_{i-1}\right),
\end{aligned}
$$

where $\mathbf{r}, \mathbf{v}$, and $\mathbf{a}$ are the position, velocity, and acceleration vectors, $\Delta t$ is the time step, and the subscripts denote the integration steps. The time step is equal to $0.5 \mathrm{fs}$, which is sufficiently small such that the total energy of the system is conserved. The temperature of the system is $94.4 \mathrm{~K}$ to make sure that Ar is in liquid state. The basic units of length, mass, and energy are set to be angstrom, the mass of one Ar molecule, and $\mathrm{eV}$, respectively. Periodic boundary conditions (PBCs) are employed in all the directions. To reduce the effect of the system size on the measurement of the particle diffusivity, the side length of the cubic simulation systems is chosen to be 5-10 times the particle diameter, depending on the particle size. This will be discussed in details later.

\section{A. Liquid viscosity}

Before measuring the particle diffusivity, the viscosity of the liquid is calculated without the solid particle in the system. The viscosity is evaluated by the Green-Kubo formula [31]

$$
\mu=\frac{1}{\forall k T} \int_{0}^{\infty}\left\langle J_{x y}(0) \cdot J_{x y}(t)\right\rangle d t,
$$

where $\forall$ is the volume, $\langle\cdot\rangle$ denotes the ensemble average, and $J_{x y}$ is the $x-y$ component stress tensor given by

$$
J_{x y}=\sum_{i=1}^{N} m_{i} v_{i}^{x} v_{i}^{y}-\sum_{i=1}^{N} \sum_{j>i}^{N} r_{i j}^{x} \frac{\partial U}{\partial r_{i j}^{y}},
$$

where $N, m$, and $v$ are the total number, mass, and velocity of liquid molecules and $i$ and $j$ are the indices of the molecules. At $94.4 \mathrm{~K}$, the viscosity of Ar obtained from MD simulations is $\mu=2.13 \pm 0.12 \times 10^{-4} \mathrm{~Pa} \mathrm{~s}$, which is in good agreement with experiments and some other MD simulations [28,32]. 


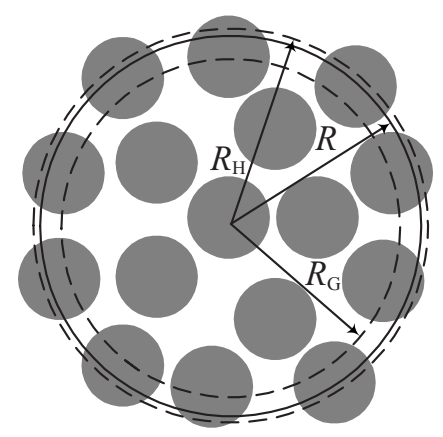

FIG. 1. Schematic relationships of radius $R$, used for constructing the particle, gyration radius $R_{G}$, and hydrodynamic radius $R_{H}$ for relatively large and spherical particles.

\section{B. Particle size and diffusivity}

The MD simulation systems involve a large number of liquid molecules and particle atoms. Numerically, it can be very expensive if the particle size is large, considering that the simulation box has to be much larger than the particle size. In this work, the largest particle is about $5 \mathrm{~nm}$ in diameter, which is quite spherical. The smallest particle only contains three atoms, which actually is a cluster and cannot be treated as a sphere. For such small particles or clusters, the hydrodynamic radius $R_{H}$ is more appropriate than the radius $R$ used to construct the particle in the simulations. The hydrodynamic radius $R_{H}$ is related to the gyration radius $R_{G}$ of the particle, where $R_{H}$ and $R_{G}$ are defined through $1 / R_{H}$ $=\left\langle\sum_{i \neq j} 1 / r_{i j}\right\rangle / N_{p}^{2}$ and $R_{G}^{2}=\left\langle\sum_{i=1}^{N_{p}}\left(r_{i}-\bar{r}\right)^{2}\right\rangle / N_{p}$, with $r_{i j}$ being the distance between a pair of particle atoms, $\bar{r}$ the mean position, and $N_{p}$ the number of atoms in the particle $[33,34]$. Since the constituent atoms of the solid particle also has a size, which is usually thought of as the collision diameter $\sigma$ in the LJ potential for liquid-particle interaction, the hydrodynamic radius $R_{H}$ should be larger than $R$ for relatively big particles, as shown in Fig. 1. Depending on the structure of the particles or clusters, the ratio of $R_{H} / R_{G}$ varies with the upper limit equal to $R_{H}=1.29 R_{G}$ [33]. The particle radii based on the various definitions are shown in Table I. By comparing the radii to $R$, it is seen that $R_{H}=1.29 R_{G}$ seems to give a reasonable prediction for the particle size. Nevertheless, we will present the diffusivity by using different particle

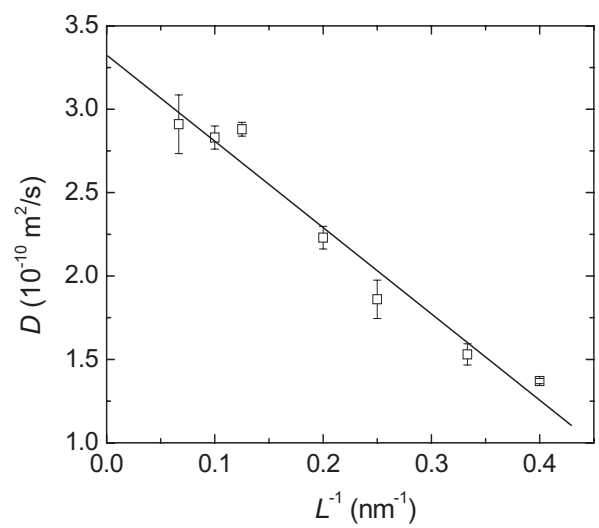

FIG. 2. The size effect of the simulation systems on the particle diffusivity. Solid line is the linear fit to the MD results.

radii to show that the definition of particle size does not affect the qualitative prediction of the critical particle size.

The diffusion coefficient of the particles is calculated by

$$
D=\lim _{t \rightarrow \infty}\left\langle(\Delta r)^{2}\right\rangle / 6 t,
$$

where $\left\langle(\Delta r)^{2}\right\rangle$ is the mean-square displacement (MSD) of the particle and $t$ is the time. In the simulations, the MSD is obtained as a function of time and the diffusivity is obtained by linearly fitting the MSD.

The PBCs in all the directions used in the simulations are equivalent to generate an infinite system, which is formed by replicating the simulation cell through rigid translations. In this infinite system, there are many solid particles, which are interacting with each another. To reduce the hydrodynamic interactions between these replicas of the particle, the simulation box should be much larger than the particle size. To understand the error caused by the finite system size, we calculate the diffusivity of a particle with diameter $d=2 R$ $=1 \mathrm{~nm}$ by changing the length $L$ of the simulation system. Figure 2 shows the diffusion coefficient $D$ of the particle as a function of $1 / L$. The liquid-particle binding energy $\varepsilon_{\mathrm{LP}}$ $=233.6 \mathrm{~K}$. It is seen that the diffusivity scales linearly with $1 / L[D=3.3-2.3 k T /(6 \pi \mu L)]$. This is in good agreement with the previous work that $D=D_{0}-2.837 k T /(6 \pi \mu L)$, where $D_{0}$ is the diffusivity predicted by the SE relation [35-37].

TABLE I. Geometric parameters and the number of molecules of the simulation systems. $N_{p}$ : number of atoms in the particle; $R$ : radius used for constructing the particles; $R_{G}$ and $R_{H}$ : gyration and hydrodynamic radii; $L / d$ : simulation box to particle size ratio; $N$ : number of fluid molecules in the system.

\begin{tabular}{rccccrr}
\hline \hline$N_{p}$ & $\begin{array}{c}R \\
(\mathrm{~nm})\end{array}$ & $\begin{array}{c}R_{G} \\
(\mathrm{~nm})\end{array}$ & $\begin{array}{c}R_{H} \\
(\mathrm{~nm})\end{array}$ & $\begin{array}{c}1.29 R_{G} \\
(\mathrm{~nm})\end{array}$ & $L / d$ & $N$ \\
\hline 3925 & 2.50 & 1.95 & 2.11 & 2.52 & 5 & 316200 \\
887 & 1.50 & 1.18 & 1.29 & 1.52 & 5 & 62000 \\
249 & 1.00 & 0.77 & 0.86 & 1.00 & 8 & 78500 \\
43 & 0.50 & 0.42 & 0.51 & 0.55 & 10 & 19600 \\
30 & & 0.38 & 0.47 & 0.49 & $>10$ & 19600 \\
10 & & 0.26 & 0.37 & 0.33 & $>10$ & 8750 \\
3 & & 0.15 & 0.25 & 0.19 & $>10$ & 3957 \\
\hline \hline
\end{tabular}



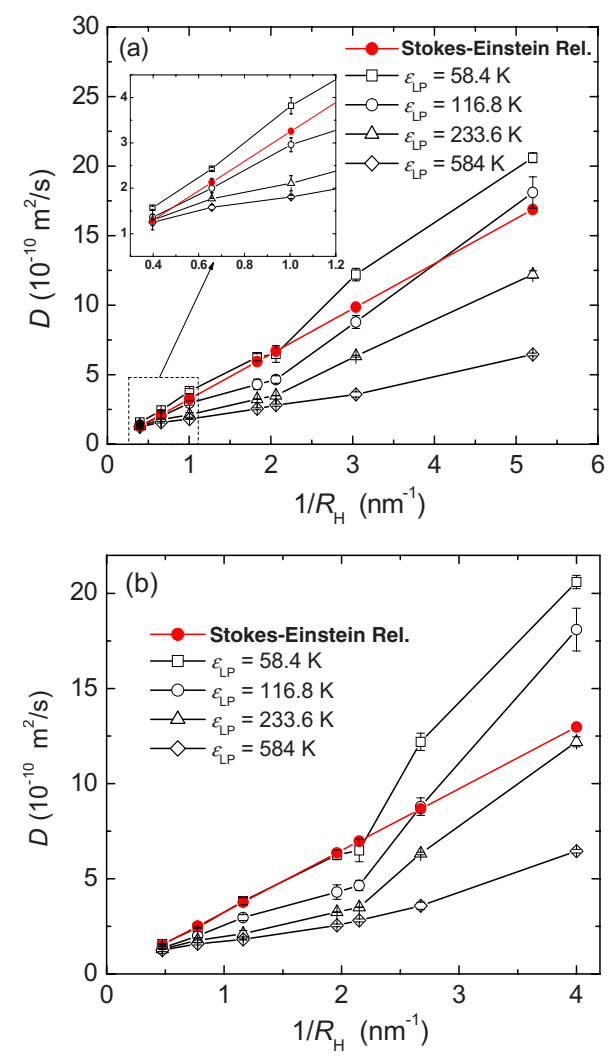

FIG. 3. (Color online) The diffusion coefficient as a function of $1 / R_{H}$ for different liquid-particle binding energy $\varepsilon_{\mathrm{LP}}$. In (a), $R_{H}$ $=1.29 R_{G}$; in (b), $R_{H}$ is defined through $1 / R_{H}=\left\langle\sum_{i \neq j} 1 / r_{i j}\right\rangle / N_{p}^{2}$. The inset in (a) is the blowup for relatively large particles.

\section{Results and discussions}

In the simulations, $L / d$ is relatively large for particles of $d \leq 2 \mathrm{~nm}(d=2 R)$. For particles of $d=3$ and $5 \mathrm{~nm}, L / d$ is set to be 5 because the systems are quite large and numerically very expensive. The values of $L / d$ and the number of fluid molecules in the corresponding simulation system are provided in Table I. As shown in Table I, there are over 300000 fluid molecules in the simulation system for the particle of $R=2.5 \mathrm{~nm}$. Such a big system is beyond the capability of our computing facility (a cluster of 20 duo-core computers of 3.0 $\mathrm{GHz}$ ) [38]. Depending on the size of the particle, 50-200 simulations with different initial conditions are used to obtain the MSD in Eq. (6). In all the simulations, the smallest system has a side length of $6 \mathrm{~nm}$ containing 3957 fluid molecules, which is sufficiently large such that the system size has little to no effect on the viscosity of the fluid [39].

To consider the effect of van der Waals force on the particle diffusion, the liquid-particle binding energy $\varepsilon_{\mathrm{LP}}$ is varied by a factor of 10 , from 58.4 to $584 \mathrm{~K}$, which covers many popular fluid-solid interactions $[10,26,40]$. Figure 3 depicts the diffusion coefficient $D$ as a function of $1 / R_{H}$ with $R_{H}$ defined through $R_{H}=1.29 R_{G}$ and $1 / R_{H}=\left\langle\Sigma_{i \neq j} 1 / r_{i j}\right\rangle / N_{p}^{2}$ in Figs. 3(a) and 3(b), respectively. The diffusivities have been corrected by the analytical factor, $\lambda=(1-2.837 R / L)^{-1}$, to account for the effect of system size on the particle diffusion. It is seen that the diffusion coefficient grows increasingly sensitive to the van der Waals force as the particle size ap- (a)

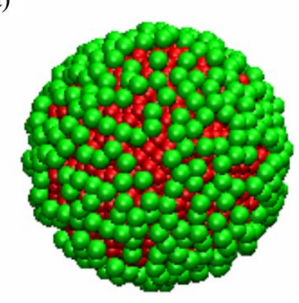

(c)

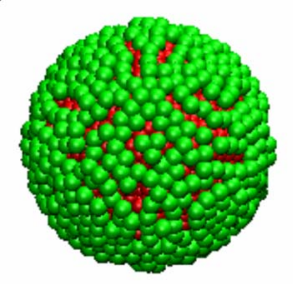

(b)

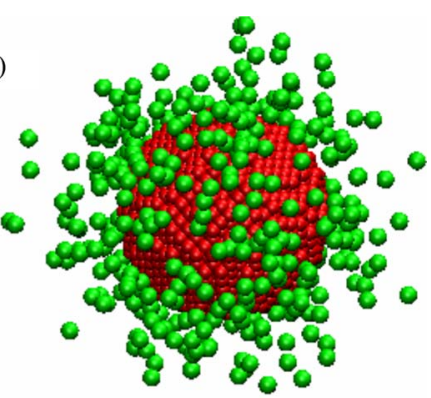

(d)

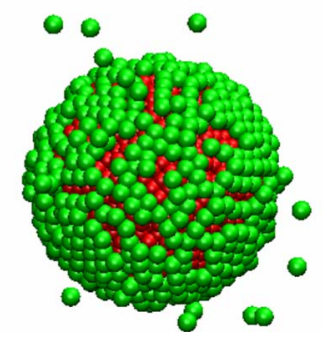

FIG. 4. (Color online) Snapshots of liquid molecules (green) around the particle (red) of $R=2.5 \mathrm{~nm}$. The binding energy $\varepsilon_{\mathrm{LP}}$ $=58.4 \mathrm{~K}$ in (a) and (b) and $\varepsilon_{\mathrm{LP}}=584 \mathrm{~K}$ in (c) and (d). (a) and (c) are the positions of some liquid molecules around the particle at a certain time. (b) and (d) are the positions of the same molecules in (a) and (c) after 200 ps.

proaches molecular scale. For relatively large particles, the van der Waals force becomes unimportant and the diffusion coefficients for all the binding energies converge to the prediction of the SE relation. It is unfortunate that we could not go further to larger particles due to the limitations of our computational facilities. Nevertheless, the trend in Fig. 3 indicates that the diffusion coefficient will be in good agreement with the SE relation if the particle size is increased further regardless of the way how the particle size is estimated and it is safe to predict that this critical particle size is of a few nanometers.

It is also noted that when the liquid-particle interaction is weak, where the slip boundary condition applies [41], the diffusion coefficients generally agree well with the classical theory regardless of the particle size, as the case of $\varepsilon_{\mathrm{LP}}$ $=58.4 \mathrm{~K}$ in Fig. 3(a). For strong binding energies, the SE relation overestimates the diffusion coefficient. This explains why the results in the literature are controversial $[13,20]$. For fluid-solid interactions, it is well known that strong interactions may cause fluid adsorption on the solid surface. The adsorbed fluid molecules can change the particle size and therefore the particle diffusivity. To understand the layering of liquid molecules on the particles, we identify some liquid molecules around a particle at a certain time and then follow these molecules for a while to find out whether these molecules are really adsorbed by the particle or not. Figure 4 demonstrates the snapshots of these molecules around the particle of $R=2.5 \mathrm{~nm}$. In Figs. 4(a) and 4(b), the liquidparticle binding energy $\varepsilon_{\mathrm{LP}}=58.4 \mathrm{~K}$. Figure $4(\mathrm{a})$ shows the initial positions of the particle and some liquid molecules in the neighborhood and Fig. 4(b) records the positions of these liquid molecules after 200 ps. It is found that the liquid mol- 
ecules are not really adsorbed by the particle for such small binding energy. However, similar snapshots for $\varepsilon_{\mathrm{LP}}=584 \mathrm{~K}$ confirm the adsorption phenomena, as illustrated in Figs. 4(c) and 4(d).

The layering of liquid molecules on the particle surface increases the particle size and reduces the diffusion coefficient of the particle. In this sense, the small diffusivity calculated from MD simulations for large binding energies is, to some extent, caused by the adsorbed liquid molecules. However, the adsorbed molecules are not the major contribution to the reduction of the particle diffusivity. This can be confirmed by the diffusivity of the particle of $R=1 \mathrm{~nm}$. If we assume that the particle is completely covered by a layer of liquid molecules for the strongest binding energy and the particle radius changes from 1 to $1.34 \mathrm{~nm}$ (the increment of $0.34 \mathrm{~nm}$ is the diameter of the liquid molecules), the diffusion coefficient of the particle will be reduced by $34 \%$, which cannot compensate the large difference between the MD simulations and SE relation, as shown in Fig. 3. Therefore, the fluid adsorption is not the major reason that leads to the reduction of particle diffusivity. This indicates that the intermolecular interactions, such as the van der Waals force, play important roles for small particle diffusion.
In the simulations, the thermal vibration of the atoms in the particle is considered, which is important for fluid-wall interaction [42]. To confirm this, we have calculated the diffusivities of rigid particles. For $\varepsilon_{\mathrm{LP}}=116.8$ and $548 \mathrm{~K}$, the diffusion coefficient of $R=0.5 \mathrm{~nm}$ particle is $1.49 \pm 0.18$ $\times 10^{-10}$ and $1.73 \pm 0.16 \times 10^{-10} \mathrm{~m}^{2} / \mathrm{s}$, respectively, if the particle is considered as a rigid body, while the diffusivity is $3.81 \pm 0.38 \times 10^{-10}$ and $2.26 \pm 0.07 \times 10^{-10} \mathrm{~m}^{2} / \mathrm{s}$ when the internal degrees of freedom are considered.

It is worth mentioning that our attention is on the critical particle size, where the SE relation breaks down. The quantitative dependence of the particle diffusivity on certain parameters is not in the scope of current work. Although we could not find exactly where the critical particle size is due to the restrictions of computing facilities, the simulation results qualitatively predict that this critical size is of a few nanometers.

\section{ACKNOWLEDGMENT}

This work was supported by the Hong Kong Innovation and Technology Fund under Grant No. GHP/035/07GD.
[1] G. G. Stokes, Proc. Cambridge Philos. Trans. 9, 8 (1851).

[2] A. Einstein, Ann. Phys. 322, 549 (1905).

[3] R. M. Mazo, Brownian Motion. Fluctuation, Dynamics, and Applications (Clarendon, Oxford, 2002).

[4] J. H. Seinfeld and S. N. Pandis, Atmospheric Chemistry and Physics: From Air Pollution to Climate Change (Wiley, New York, 1997).

[5] J. A. Griffiths and D. M. Heyes, Langmuir 12, 2418 (1996).

[6] F. Reif, Fundamentals of Statistical and Thermal Physics (McGraw-Hill, Singapore, 1985).

[7] F. Eirich, Kolloid-Z. 74, 276 (1936).

[8] Z. Li and H. Wang, Phys. Rev. Lett. 95, 014502 (2005).

[9] Z. Li and H. Wang, Phys. Rev. E 68, 061206 (2003).

[10] Z. Li and H. Wang, Phys. Rev. E 68, 061207 (2003).

[11] B. J. Alder, D. M. Gass, and T. E. Wainwright, J. Chem. Phys. 53, 3813 (1970).

[12] L. Bocquet, J. P. Hansen, and J. Piasecki, J. Stat. Phys. 76, 527 (1994).

[13] M. Vergeles, P. Keblinski, J. Koplik, and J. R. Banavar, Phys. Rev. E 53, 4852 (1996).

[14] L. G. Longsworth, J. Am. Chem. Soc. 74, 4155 (1952).

[15] F. Ould-Kaddour and J.-L. Barrat, Phys. Rev. A 45, 2308 (1992).

[16] F. Ould-Kaddour and D. Levesque, Phys. Rev. E 63, 011205 (2000).

[17] R. Walser, A. E. Mark, and W. F. van Gunsteren, Chem. Phys. Lett. 303, 583 (1999).

[18] D. M. Heyes, M. J. Nuevo, and J. J. Morales, J. Chem. Soc., Faraday Trans. 94, 1625 (1998).

[19] J. R. Schmidt and J. L. Skinner, J. Phys. Chem. B 108, 6767 (2004).

[20] J. R. Schmidt and J. L. Skinner, J. Chem. Phys. 119, 8062
(2003).

[21] R. O. Sokolovskii, M. Thachuk, and G. N. Patey, J. Chem. Phys. 125, 204502 (2006).

[22] M. Sharma and S. Yashonath, J. Phys. Chem. B 110, 17207 (2006).

[23] F. Ould-Kaddour and D. Levesque, J. Chem. Phys. 127, 154514 (2007).

[24] J. Liu, D. Cao, and L. Zhang, J. Phys. Chem. C 112, 6653 (2008).

[25] A. Tuteja, M. E. Mackay, S. Narayanan, S. Asokan, and M. S. Wong, Nano Lett. 7, 1276 (2007).

[26] H. Hippler, J. Troe, and H. J. Wendelken, J. Chem. Phys. 78, 6709 (1983).

[27] J. O. Osburn, J. A. Stitzell, and R. E. Peterson, J. Appl. Physiol. 27, 624 (1969).

[28] G. A. Fernandez, J. Vrabec, and H. Hasse, Fluid Phase Equilib. 221, 157 (2004).

[29] F. Cleri and V. Rosato, Phys. Rev. B 48, 22 (1993).

[30] Z. Li and G. Drazer, Phys. Fluids 18, 117102 (2006).

[31] J. M. Haile, Molecular Dynamics Simulation (John Wiley and Sons, New York, 1992).

[32] D. G. Naugle, J. H. Lunsford, and J. R. Singer, J. Chem. Phys. 45, 4669 (1966).

[33] P. Wiltzius, Phys. Rev. Lett. 58, 710 (1987).

[34] F. Perrin, J. Phys. Radium 7, 1 (1936).

[35] H. Hasimoto, J. Fluid Mech. 5, 317 (1959).

[36] A. S. Sangani and A. Acrivos, Int. J. Multiphase Flow 8, 343 (1982).

[37] V. Lobaskin and B. Dunweg, New J. Phys. 6, 54 (2004); P. Ahlrichs and B. Dunweg, J. Chem. Phys. 111, 8225 (1999); B. Dunweg and K. Kremer, ibid. 99, 6983 (1993). 
[38] It is noted that most of the CPU time are spent for calculating the fluid-particle interactions. The tight-binding potential for the particle is not the major reason for time consumption. If the LJ potential is used for the particle, for the system with the largest particle, the CPU time can only be reduced by about $7 \%$.
[39] I. C. Yeh and G. Hummer, J. Phys. Chem. B 108, 15873 (2004).

[40] J. O. Hirschfelder, C. F. Curtiss, and R. B. Bird, Molecular Theory of Gases and Liquids (Wiley, New York, 1954).

[41] C. Liu and Z. Li, Phys. Rev. E 80, 036302 (2009).

[42] Z. Li and L. Hong, J. Chem. Phys. 127, 074706 (2007). 